\title{
Computational issues in fault detection filter design
}

\author{
Andras Varga \\ German Aerospace Center \\ DLR - Oberpfaffenhofen \\ Institute of Robotics and System Dynamics \\ D-82234 Wessling, Germany \\ Andras.Varga@dlr.de
}

\begin{abstract}
We discuss computational issues encountered in the design of residual generators for dynamic inversion based fault detection filters. The two main computational problems in determining a proper and stable residual generator are the computation of an appropriate leftinverse of the fault-system and the computation of coprime factorizations with proper and stable factors. We discuss numerically reliable approaches for both of these computations relying on matrix pencil approaches and recursive pole assignment techniques for descriptor systems. The proposed computational approach to design fault detection filters is completely general and can easily handle even unstable and/or improper systems.
\end{abstract}

\section{Introduction}

Consider the system described by the input-output relations

$$
\mathbf{y}(\lambda)=G_{p}(\lambda) \mathbf{u}(\lambda)+G_{f}(\lambda) \mathbf{f}(\lambda)+G_{d}(\lambda) \mathbf{d}(\lambda),
$$

where $\mathbf{y}(\lambda), \mathbf{u}(\lambda), \mathbf{f}(\lambda)$, and $\mathbf{d}(\lambda)$ are transformed vectors of the $p$-dimensional system output vector $y(t)$, $m$-dimensional plant input vector $u(t), q$-dimensional fault signal vector $f(t)$, and $r$-dimensional disturbance vector $d(t)$, respectively, and where $G_{p}(\lambda), G_{f}(\lambda)$ and $G_{d}(\lambda)$ are the transfer-function matrices (TFMs) from the plant inputs to outputs, fault signals to outputs, and disturbances to outputs, respectively. According to the system type, $\lambda$ is either the complex variable $s$ appearing in the Laplace transform in the case of a continuous-time system or the variable $z$ appearing in the $Z$-transform in the case of a discrete-time system.

One of the standard approaches to design residual generators is based on computing dynamic inversion based fault detection filters $[5,6,10,12,8,3,9]$. It is wellknown (see for example $[5,6]$ ) that, if: 1) $G_{p}(\lambda)$ is stable; 2) $\left[G_{f}(\lambda) G_{d}(\lambda)\right]$ is left-invertible; 3) $G_{f}(\lambda)$ is minimum-phase; and 4) $G_{f}(\lambda)$ has no infinite zeros, then a physically realizable fault detection filter can be determined as

$$
\mathbf{r}(\lambda)=Q(\lambda)\left(\mathbf{y}(\lambda)-G_{p}(\lambda) \mathbf{u}(\lambda)\right),
$$

where $Q(\lambda)=G_{f}^{+}(\lambda)$ is a stable proper left-inverse of $G_{f}(\lambda)$ and $Q(\lambda) G_{d}(\lambda)=0$. For zero initial conditions, this detector has the nice property that $r(t)=f(t)$, that is, the residual is equal to the fault signal.

In the case of an unstable or improper plant a modified detector can be used based on a left coprime factorization of $G_{p}(\lambda)$ in the form

$$
G_{p}(\lambda)=M_{p}^{-1}(\lambda) N_{p}(\lambda)
$$

where $N_{p}(\lambda)$ and $M_{p}(\lambda)$ are proper, stable and mutually coprime rational matrices. Provided $\left[G_{f}(\lambda) G_{d}(\lambda)\right]$ and $G_{f}(\lambda)$ further satisfy conditions $2), 3$ ) and 4$)$, and additionally $M_{p}(\lambda)$ is biproper and minimum phase, then we can use the residual generator $[5,6]$

$$
\mathbf{r}(\lambda)=Q(\lambda)\left(M_{p}(\lambda) \mathbf{y}(\lambda)-N_{p}(\lambda) \mathbf{u}(\lambda)\right)
$$

where $Q(\lambda)=G_{f}^{+}(\lambda) M_{p}^{-1}(\lambda)$ is a stable proper leftinverse of $M_{p}(\lambda) G_{f}(\lambda)$ and $Q(\lambda) M_{p}(\lambda) G_{d}(\lambda)=0$. For a stable plant, $M_{p}(\lambda)=I$ and we recover the simple case (1). Note that the conditions on $M_{p}(\lambda)$ (minimumphase, biproper) are usually not satisfied, so a modification of the above detector is necessary to ensure physical realizability.

In the most general setting, considered in this paper, $M_{p}(\lambda) G_{f}(\lambda)$ can have infinite zeros and/or can have non-minimum phase. In the first case, the left-inverse is not anymore proper, while in the second case no stable left-inverse exists. A common approach to overcome these difficulties $[10,9]$ is to determine a diagonal matrix $M(\lambda)$ such that $Q(\lambda):=M(\lambda) G_{f}^{+}(\lambda) M_{p}^{-1}(\lambda)$ is a proper and stable TFM. With this modification, a physically realizable residual generator of the form (2) can be used. It is always possible to determine $M(\lambda)$ to additionally ensure (e.g., by means of a diagonal scaling) that $r(t) \rightarrow f(t)$ for $t \rightarrow \infty$. Alternatively, instead of a diagonal $M(\lambda)$, we can determine from a left coprime factorization of $G_{f}^{+}(\lambda) M_{p}^{-1}(\lambda)=M^{-1}(\lambda) Q(\lambda)$ a 
least McMillan degree $M(\lambda)$ such that $Q(\lambda)$ is proper and stable. The resulting $Q(\lambda)$ has the same McMillan degree as $G_{f}^{+}(\lambda) M_{p}^{-1}(\lambda)$ which is in general lower than when employing a diagonal $M(\lambda)$. For the resulting residual generator we can still achieve a static decoupling (i.e., $r(t) \rightarrow f(t)$ for $t \rightarrow \infty$ ) by determining a constant (non-diagonal) matrix $R$ such that $R Q\left(\lambda_{s}\right) M_{p}\left(\lambda_{s}\right) G_{f}\left(\lambda_{s}\right)=I$, where $\lambda_{s}=0$ for continuous-time system and $\lambda_{s}=1$ for a discrete-time system. Moreover, by suitably choosing $M(\lambda)$, an arbitrary fast dynamics of the detector can be achieved. For example, in the discrete-time case a finite-time deadbeat type detector filter $M(z)$ can be determined, which corresponds to the so-called polynomial residual generator discussed in [10].

The algorithms underlying the design of residual generators require the manipulation of rational matrices. For low dimensional systems, this is possible to some extent by using symbolic manipulation software as provided by tools like Maple or Mathematica. However for large order systems, symbolic computation is not anymore applicable because of tremendous manipulation efforts, and therefore the usage of numerical algorithms is the only possible option. The need to address the numerical issues to solve computational problems encountered in designing fault detection and isolation filters has been already recognized in [3, page 219]. Our main motivation for this paper was to bring into the attention of fault detection experts some of the latest developments in computational algorithms for manipulation of rational matrices. By using recently developed numerically reliable state space algorithms (proposed mainly in mathematical journals), many of computational problems in the fault detection field can be addressed for high dimensional systems.

In this paper we discuss in some details the two main computational problems encountered in determining a residual generator, namely, the computation of a leftinverse with special properties and the determination of rational factorizations by using coprime factorization techniques. The computation of the left-inverse is based on recently developed pencil algorithms [18] which allow to obtain the inverse by only manipulating the matrices of a state space realization. The inverse is obtained in a descriptor system form without any need to invert a constant or polynomial/rational matrix. Stable and proper inverses are easy to compute when they exist. The computation of proper and stable fractional factorizations can be done by using coprime factorization techniques based on recursive pole assignment [16]. The resulting denominator factors have least McMillan degrees. Using the numerically reliable inversion and factorization algorithms described in this paper, the fault detection filter design problem can be solved in its most general setting. Several standard functions available in a recently developed Descriptor
System Toolbox for MATLAB [17] allow a straightforward implementation of the underlying computations.

\section{Computation of left-inverses}

For the design of residual generators, we have to compute the left-inverse $G^{+}(\lambda)$ of a full column rank rational matrix $G(\lambda)=M_{p}(\lambda) G_{f}(\lambda)$ which additionally must satisfy the condition $G^{+}(\lambda) M_{p}(\lambda) G_{d}(\lambda)=0$. To solve this problem we form the extended TFM $G_{e}(\lambda)=M_{p}(\lambda)\left[G_{f}(\lambda) G_{d}(\lambda)\right]$ and assume $G_{e}(\lambda)$ has full column rank (i.e., is left-invertible). Let $G_{e}^{+}(\lambda)$ be a left-inverse of $G_{e}(\lambda)$ partitioned row-wise in accordance with the column-wise structure of $G_{e}(\lambda)$

$$
G_{e}^{+}(\lambda)=\left[\begin{array}{c}
G_{f}^{+}(\lambda) \\
G_{d}^{+}(\lambda)
\end{array}\right] M_{p}^{-1}(\lambda)
$$

From $G_{e}^{+}(\lambda) G_{e}(\lambda)=I_{q+r}$, we obtain

$$
G_{f}^{+}(\lambda) G_{f}(\lambda)=I_{q}, \quad G_{f}^{+}(\lambda) G_{d}(\lambda)=0
$$

thus the resulting $G^{+}(\lambda)=G_{f}^{+}(\lambda) M_{p}^{-1}(\lambda)$ fulfils the two basic requirements formulated above. In this way, the first step in determining the filter matrix $Q(\lambda)$ can be done by computing the left-inverse of the extended matrix $G_{e}(\lambda)$. The case of non-full column rank $G_{d}(\lambda)$ (see $[6]$ ), can be handled by simply eliminating the linearly dependent columns of this TFM or by computing a more general type of inverse (a so-called $(1,2)$-inverse) of $G_{e}(\lambda)$ using the method of [18].

In what follows, we only discuss the computation of a left-inverse of a left-invertible $p \times q$ TFM $G(\lambda)$ with a minimal descriptor realization

$$
\begin{aligned}
E \lambda x(t) & =A x(t)+B \nu(t) \\
\xi(t) & =C x(t)+D \nu(t)
\end{aligned}
$$

satisfying

$$
G(\lambda)=C(\lambda E-A)^{-1} B+D
$$

According to the system type, $\lambda$ also represents either the differential operator $\lambda x(t)=\dot{x}(t)$ in the case of a continuous-time system or the advance operator $\lambda x(t)=x(t+1)$ in the case of a discrete-time system. Note that for most practical applications $G(\lambda)$ is a proper TFM, thus we can always choose a realization such that $E=I$. However, for the sake of generality, we will keep $E$ in our system descriptions, and we only assume that the pencil $A-\lambda E$ is regular. In this way, we will cover also the case of singular systems addressed, for example in [22]. Throughout the paper, we will use the bold-notation $\mathbf{G}$ to denote a descriptor system $(A-\lambda E, B, C, D)$ having the TFM $G(\lambda)$.

Let $S(\lambda)$ be the system pencil associated to the system G

$$
S(\lambda)=\left[\begin{array}{c|c}
A-\lambda E & B \\
\hline C & D
\end{array}\right] .
$$


Provided $G(\lambda)$ has full-column rank, a left-inverse $G^{+}(\lambda)$ can be computed using the following straightforward formula

$$
G^{+}(\lambda)=\left[\begin{array}{ll}
0 & I_{q}
\end{array}\right] S^{+}(\lambda)\left[\begin{array}{c}
0 \\
I_{p}
\end{array}\right],
$$

where $S^{+}(\lambda)$ is a left-inverse of $S(\lambda)$. By using this formula, the computation of the left-inverse of $G(\lambda)$ can be accomplished by computing the left-inverse of the associated full-column rank system pencil $S(\lambda)$.

We will need the following result to compute leftinverses (see e.g., [1]):

Lemma 1 Let $R(\lambda)$ be a rational matrix of full column rank $k$ and let $P$ a permutation matrix such that

$$
P R(\lambda)=\left[\begin{array}{l}
R_{1}(\lambda) \\
R_{2}(\lambda)
\end{array}\right]
$$

where rank $R_{1}(\lambda)=k$. Then, a left-inverse of $R(\lambda)$ is

$$
R^{+}(\lambda)=\left[\begin{array}{ll}
R_{1}^{-1}(\lambda) & 0
\end{array}\right] P .
$$

The computation of $S^{+}(\lambda)$ can be done by reducing $S(\lambda)$ to an appropriate Kronecker-like form from which a full row regular sub-pencil can be easily separated. Let $Q$ and $Z$ be orthogonal matrices to reduce $S(\lambda)$ to the particular Kronecker-like form [15]

$$
\begin{aligned}
\bar{S}(\lambda) & :=Q S(\lambda) Z=\left[\frac{\bar{S}_{1}(\lambda)}{\bar{S}_{2}}\right]= \\
& =\left[\begin{array}{cc}
A_{r e g}-\lambda E_{r e g} & * \\
0 & A_{l}-\lambda E_{l} \\
\hline 0 & C_{l}
\end{array}\right]
\end{aligned}
$$

where the regular part $A_{r e g}-\lambda E_{r e g}$ contains the finite and infinite system zeros, and the pair $\left(C_{l}, A_{l}-\lambda E_{l}\right)$ is observable with $E_{l}$ nonsingular. Since

$$
\operatorname{rank} \bar{S}(\lambda)=\operatorname{rank} \bar{S}_{1}(\lambda),
$$

by applying Lemma 1 with $R(\lambda)=\bar{S}(\lambda), k=n+q$ and $P=I_{n+q}$, we obtain a left-inverse of $G(\lambda)$ with a descriptor system representation

$$
\mathbf{G}^{+}:=(\bar{A}-\lambda \bar{E}, \bar{B}, \bar{C}, 0),
$$

where

$$
\begin{gathered}
\bar{A}-\lambda \bar{E}:=\bar{S}_{1}(\lambda), \quad\left[\frac{\bar{B}}{\bar{D}}\right]:=Q\left[\begin{array}{c}
0 \\
I_{p}
\end{array}\right] \\
\bar{C}:=\left[0-I_{q}\right] Z
\end{gathered}
$$

The eigenvalues of the inverse are

$$
\Lambda(\bar{A}, \bar{E})=\Lambda_{\text {fixed }} \cup \Lambda_{\text {spurious }},
$$

where $\Lambda_{\text {fixed }}=\Lambda\left(A_{\text {reg }}, E_{\text {reg }}\right)$ are the eigenvalues of the regular part an thus contains the system zeros, and $\Lambda_{\text {spurious }}=\Lambda\left(A_{l}, E_{l}\right)$ are the finite "spurious" zeros originating from the row singularity of $G(\lambda)$.

While the system zeros are always among the poles of the inverse, the spurious poles can be arbitrarily modified. To show this, consider the transformation matrix $U$ of the form

$$
U=\left[\begin{array}{cc|c}
I & O & O \\
0 & I & K \\
\hline O & O & I
\end{array}\right]
$$

Then the transformed system pencil is given by

$$
\begin{aligned}
& \widehat{S}(\lambda):=U \bar{S}(\lambda)=\left[\frac{\widehat{S}_{1}(\lambda)}{\widehat{S}_{2}}\right] \\
& * \\
&= {\left[\begin{array}{cc}
A_{\text {reg }}-\lambda E_{r e g} & * \\
0 & A_{l}+K C_{l}-\lambda E_{l} \\
\hline 0 & C_{l}
\end{array}\right] }
\end{aligned}
$$

The left-inverse is defined now by

$$
\mathbf{G}^{+}:=(\widehat{A}-\lambda \widehat{E}, \widehat{B}, \widehat{C}, 0),
$$

where

$$
\begin{aligned}
\widehat{A}-\lambda \widehat{E} & :=\widehat{S}(\lambda), \\
{\left[\begin{array}{c}
\widehat{B} \\
\widehat{D}
\end{array}\right] } & :=U Q\left[\begin{array}{c}
0 \\
I_{p}
\end{array}\right], \\
\widehat{C} & :=\left[0-I_{q}\right] Z .
\end{aligned}
$$

When using the transformed pencil $\widehat{S}(\lambda)$, the spurious poles of the left-inverse are

$$
\Lambda_{\text {spurious }}=\Lambda\left(A_{l}+K C_{l}, E_{l}\right) .
$$

To obtain a left-inverse which requires fewer manipulation later, we can assign the spurious poles to lie in a "good" domain $\mathbb{C}_{g}$ of the complex plane, by choosing $K$ such that $\Lambda\left(A_{l}+K C_{l}, E_{l}\right) \subset \mathbb{C}_{g}$. For example, we can choose $\mathbb{C}_{g}=\mathbb{C}^{-}$, where $\mathbb{C}^{-}$is the appropriate stability domain for poles (i.e., the open left half complex plane in the case of a continuous-time system or the interior of the unit circle in the case of a discretetime system.) However, in the discrete-time case, we can also choose $\mathbb{C}_{g}=\{0\}$, thus assigning all spurious poles in the origin. This is convenient when we want to design generator filters with a dead-beat behavior. To assign the spurious poles, the pole assignment algorithm for descriptor systems based on a generalized Schur method can be employed [14].

The main properties of the computed left-inverse $G^{+}(\lambda)$ can be deduced from the Kronecker-structure of the system matrix $S(\lambda)$. The left-inverse will be proper if no infinite zeros are present, that is, all infinite eigenvalues of the matrix pair $\left(A_{r e g}, E_{r e g}\right)$ are simple (i.e., 
non-dynamical). Additionally, a stable left-inverse can be determined if the system is minimum-phase, that is, the pair $\left(A_{r e g}, E_{r e g}\right)$ has all finite generalized eigenvalues in $\mathbb{C}^{-}$. The dynamical order (i.e., the McMillan degree) of the resulting left-inverse is the sum of the number of zeros $n_{z}$ (finite and infinite) and the number $n_{s}$ of spurious poles.

While $n_{z}$ is fixed, $n_{s}$ depends generally on the procedure used to construct the left-inverse. Thus, an interesting aspect is the computation of left-inverses with least McMillan degree. This aspect can be addressed by our approach in the following way. We can choose $K$ to make some of spurious poles of $G^{+}(\lambda)$ uncontrollable or unobservable. For example, choosing $\widehat{K}:=\left[0 K^{T}\right]^{T}$ such that a maximum number of spurious poles corresponding to the pair $(\widehat{A}+\widehat{K} \widehat{C}-\lambda \widehat{E}, \widehat{B})$ becomes uncontrollable is essentially a descriptor system generalization of the dual problem of finding an $(A, B)-$ invariant subspace of least dimension which contains a given subspace [11]. Finding $\widehat{K}$ and $\widehat{L}$ such that the pair $\left(\widehat{A}+\widehat{K} \widehat{C}-\lambda \widehat{E}, \widehat{C}+\widehat{L} \widehat{S}_{2}\right)$ has a maximum number of unobservable poles is the dual of the descriptor formulation of the minimum cover design problem of [11]. For both these computations reliable algorithms need to be developed. Although the least-order inverses are possibly unstable, still they could be useful by leading to lower dynamical complexity of residual generators. A much more difficult problem is to compute least McMillan degree inverses with the additional constraint that the spurious poles lie in a given domain $\mathbb{C}_{g}$. As far as we know, there is no complete solution of this problem, this being equivalent to the difficult question of stabilizing a linear system via constant gain output feedback (see also [20]).

\section{Computation of fractional representations}

The second main computational problem in designing residual generators is the computation of a fractional representations with proper and stable factors of a given rational matrix $G(\lambda)$, which is possibly unstable (i.e., with some poles in $\mathrm{C}^{+}$) and/or improper (i.e., with some poles at infinity). Here $G(\lambda)$ stays either for $G_{p}(\lambda)$, the TFM of the plant, or for the product $G_{f}^{+}(\lambda) M_{p}^{-1}(\lambda)$ (see Section 1). The computational problem is to determine a left coprime factorization (LCF) of $G(\lambda)$ as

$$
G(\lambda)=M^{-1}(\lambda) N(\lambda)
$$

where $M(\lambda)$ and $N(\lambda)$ are stable and proper TFMs. An important aspect in designing lower complexity detectors is the computation of a factorization with least McMillan degree for $M(\lambda)$. A second problem which we address is how to compute a fractional representation of form (7) by employing a diagonal $M(\lambda)$ of least McMillan degree.
To compute a proper coprime factorization for a given TFM $G(\lambda)$, numerical algorithms have been proposed in [16]. Specifically, the dual of Algorithm PRRCF2 of [16] computes an $M(\lambda)$ with least McMillan degree such that $M(\lambda) G(\lambda)$ is proper and has poles in a given "good" domain $\mathbb{C}_{g}$. This algorithm is based on a descriptor realization of $G(\lambda)$ as in (3) satisfying (4). If $G(\lambda)$ is proper (e.g., $E=I$ ), the resulting order of the denominator $M(\lambda)$ is the number of unstable poles of $G(\lambda)$. By using an appropriate state feedback, all unstable poles are moved to arbitrary positions in $\mathbb{C}_{g}$, while the rest of poles of $G(\lambda)$ (lying in $\mathbb{C}_{g}$ ) are preserved in $N(\lambda)$. The pole assignment for finite poles is done recursively using the generalized real Schur form of the pair $(A, E)$ (the real Schur form of $A$ if $E=I)$. In the case of poles at infinity, a preliminary state feedback is used to move them to finite positions. In this case, a preliminary spectral separation of the eigenvalues of the pencil $A-\lambda E$ (finite-infinite, $\mathbb{C}_{g}$-stable-- $\mathbb{C}_{g}$-unstable) by orthogonal reordering of the generalized Schur form of the pair $(A, E)$ (see Algorithm 2 in [16]), is performed. The McMillan degree of the resulting $M(\lambda)$ is the sum of numbers of poles at infinity and poles lying outside $\mathbb{C}_{g}$. An advantage of using Algorithm PRRCF2 of [16] is that the original descriptor realization needs not be impulse-observable (i.e., can have unobservable poles at infinity) and $\mathbb{C}_{g}$-detectable (i.e., can have finite unobservable $\mathbb{C}_{g}$-unstable poles) (see [4] for precise definitions of these concepts).

The above approach can also be used to determine a fractional representation of form (7) with proper and stable factors, and with $M(\lambda)$ diagonal. This can be done by solving $q$ independent LCF problems for singleoutput systems corresponding to each of $q$ rows of $G(\lambda)$. Assuming $G_{i}(\lambda)$ is the $i$-th row of $G(\lambda)$, we can compute the LCF

$$
G_{i}(\lambda)=\frac{N_{i}(\lambda)}{m_{i}(\lambda)}
$$

where both $m_{i}(\lambda)$ and $N_{i}(\lambda)$ are proper, stable and mutually coprime. The scalar transfer-function $m_{i}(\lambda)$ and the resulting $N_{i}(\lambda)$ are the $i$-th diagonal element of $M(\lambda)$ and the $i$-th row of $N(\lambda)$, respectively. One distinctive feature of these single-output factorization problems is that each $G_{i}(\lambda)$, arising form the overall descriptor realization of $G(\lambda)$, is generally described by a non-minimal (usually non-observable) descriptor realization. This aspect is handled automatically when employing the Algorithm PRRCF2 of [16]. Since each of resulting $m_{i}(\lambda)$ has least order McMillan degree, the resulting diagonal matrix $M(\lambda)$ has least order McMillan degree as well. Note however, that in general, the least achievable McMillan degree for a diagonal $M(\lambda)$ is greater than the least achievable McMillan degree for a non-diagonal denominator.

One final aspect to be discussed is the computation of a minimal realization of $N(\lambda)=M(\lambda) G(\lambda)$ from 
given $M(\lambda)$ and $G(\lambda)$. This problem arises, for example, when determining a diagonal $M(\lambda)$, with the diagonal entries resulting from independent LCFs of singleoutput systems. Since the resulting $N(\lambda)$ is proper and $\mathrm{C}_{g}$-stable, it has a standard state-space representation with only $\mathrm{C}_{g}$-stable eigenvalues. This minimal realization can be constructed in two ways. For example, we can compute an irreducible descriptor representation of $M(\lambda) G(\lambda)$ by forming explicitly the realization of the product $M(\lambda) G(\lambda)$ and applying the orthogonal staircase algorithms of [13] to remove successively the uncontrollable infinite and $\mathbf{C}_{g}$-unstable parts. Alternatively, we can assume that both realizations of $M(\lambda)$ and $G(\lambda)$ have state-space matrices in real Schur form or generalized real Schur form. Thus we can construct the descriptor realization of $M(\lambda) G(\lambda)$ with the descriptor pair in a generalized real Schur form too. Since all infinite and $\mathbb{C}_{g}$-unstable eigenvalues are uncontrollable, they can be removed by just reordering the generalized real Schur form such that all these eigenvalues are in the trailing positions on the diagonal. By applying the orthogonal transformation matrices to the input and output matrices, the rows of the input matrix corresponding to the trailing eigenvalues must necessarily be zero. Thus the descriptor system is in a Kalmanlike controllability form where the minimal part can be easily recovered.

\section{Designing minimal order detectors}

One interesting aspect for practical applications is the computation of minimal order detectors. The detector

(2) is implemented as a system with inputs $u(t)$ and $y(t)$, and output $r(t)$, and has the TFM

$$
R(\lambda)=Q(\lambda)\left[M_{p}(\lambda)-N_{p}(\lambda)\right]
$$

So, it is often the case that the order of the detector is at least the order of the system. This is however not always true, as revealed by the following simple example [7]:

$$
G_{p}(s)=\left[\begin{array}{c}
\frac{1}{s+1} \\
\frac{1}{(s+1)^{2}}
\end{array}\right], \quad G_{f}(s)=\left[\begin{array}{l}
0 \\
1
\end{array}\right], \quad G_{d}=0
$$

A minimal order left-inverse of $G_{f}(s)$ is $Q(s)=\left[\begin{array}{ll}0 & 1\end{array}\right]$, which leads to a second order detector

$$
R(s)=\left[\begin{array}{lll}
0 & 1 & -\frac{1}{(s+1)^{2}}
\end{array}\right]
$$

However, by choosing a first order left-inverse

$$
Q(s)=\left[\begin{array}{ll}
-\frac{1}{s+1} & 1
\end{array}\right]
$$

the corresponding detector is of first order

$$
R(s)=\left[\begin{array}{lll}
-\frac{1}{s+1} & 1 & 0
\end{array}\right]
$$

One possibility to determine a least order $R(\lambda)$ is to solve the following minimal design problem: find a least McMillan degree solution $R(\lambda)$ of the linear equation

$$
R(\lambda)\left[\begin{array}{ccc}
G_{f}(\lambda) & G_{d}(\lambda) & G_{p}(\lambda) \\
O & O & I_{m}
\end{array}\right]=\left[\begin{array}{lll}
I_{q} & O & O
\end{array}\right]
$$

This equation arises by imposing for a detector of the general form

$$
\mathbf{r}(\lambda)=R(\lambda)\left[\begin{array}{l}
\mathbf{y}(\lambda) \\
\mathbf{u}(\lambda)
\end{array}\right]
$$

the condition that $r(t)=f(t)$ for all $d(t)$ and $u(t)$ for zero initial conditions. To solve the above equation, the minimum degree algorithm of [19] can be considered as basis for a possible numerical approach using polynomial techniques. Alternatively, we can try to use a state space based approach by computing a least order stable left-inverse $G^{+}(\lambda)$ of

$$
G(\lambda)=\left[\begin{array}{ccc}
G_{f}(\lambda) & G_{d}(\lambda) & G_{p}(\lambda) \\
O & O & I_{m}
\end{array}\right]
$$

and determine $R(\lambda)$ as the first $q$ rows of $G^{+}(\lambda)$. In fact, determining a least order $R(\lambda)$ is part of the computation of the left-inverse $G^{+}(\lambda)$, and can be explicitly addressed by the dual output-nulling algorithm mentioned in Section 2. If the resulting $R(\lambda)$ is not proper or is unstable, the factorization techniques of previous section can be employed to determine a modified detector.

For the above example, we have

$$
G(s)=\left[\begin{array}{cc}
0 & \frac{1}{s+1} \\
1 & \frac{1}{(s+1)^{2}} \\
0 & 1
\end{array}\right]
$$

and by employing the pencil algorithm of Section 2 we determine a first order stable left-inverse of least McMillan degree

$$
G^{+}(\lambda)=\left[\begin{array}{ccc}
-\frac{s+3}{2(s+1)} & 1 & \frac{1}{2(s+1)} \\
0 & 0 & 1
\end{array}\right]
$$

Thus, a least order stable detector for the system (8) has the TFM

$$
R(s)=\left[\begin{array}{lll}
-\frac{s+3}{2(s+1)} & 1 & \frac{1}{2(s+1)}
\end{array}\right]
$$

\section{Conclusions}

We presented numerically reliable algorithms to solve two basic computational problems encountered in the 
design of fault detection and isolation filters: the computation of left-inverses of rational matrices and the computation of stable proper coprime factorizations. Using these algorithms, the fault detection and isolation problem with disturbance rejection can be solved in the most general setting. One problem which still needs a satisfactory numerical solution is the computation of minimal order detectors. This problem has been also addressed in [7] via minimal polynomial basis solutions, but without providing a complete solution to the fault detection and isolation problem. We have shown that a least McMillan degree detector can be computed by solving a minimum degree design problem. We also indicated (without a proof) that a possible solution to this problem is to compute a left-inverse of least McMillan degree of a certain rational matrix.

For the proposed computational approach to design residual generators, all basic numerical software is available in the Descriptor Toolbox for Matlab [17], as for example, computation of left-inverses with prescribed spectrum for spurious poles, computation of poles and zeros of descriptor systems, reduction to Kronecker-like forms, determination of minimal realizations, proper coprime factorization, etc. The basic computational tools in this toolbox are several mex-files, representing MATLAB interfaces to powerful and numerically robust Fortran subroutines available in the control and systems library SLICOT [2].

Acknowledgment. The author thanks Professor János Gertler for reading the manuscript and providing helpful suggestions to improve the paper.

\section{References}

[1] A. Ben Israel and T. N. E. Greville. Some topics in generalized inverses of matrices. In M. Z. Nashed (Ed.), Generalized Inverses and Applications, pp. 125147, Academic Press, New York, 1976.

[2] P. Benner, V. Mehrmann, V. Sima, S. Van Huffel, and A. Varga. SLICOT - a subroutine library in systems and control theory. In B. N. Datta (Ed.), Applied and Computational Control, Signals and Circuits, vol. 1, pp. 499-539, Birkhäuser, 1999.

[3] J. Chen and R. J. Patton. Robust Model-Based Fault Diagnosis for Dynamic Systems. Kluwer Academic Publisheres, London, 1999.

[4] L. Dai. Singular Control Systems, Lect. Notes Contr. Inf. Scie., vol. 118, Springer Verlag, New York, 1989.

[5] X. Ding and P. M. Frank. Fault detection via factorization. Systems \& Control Lett., 14:431-436, 1990.

[6] P. M. Frank and X. Ding. Frequency domain approach to optimally robust residual generation and evaluation for model-based fault diagnosis. Automatica, 30:789-804, 1994.

[7] E. Frisk. Order of residual generators - bounds and algorithms. Prepr. IFAC Symp. SAFEPROCESS'2000, Budapest, Hungary, pp. 599-604, 2000.

[8] J. Gertler. Fault Detection and Diagnosis in Engineering Systems. Marcel Dekker, New York, 1998.

[9] J. Gertler. Designing dynamic consistency relation for fault detection and isolation. Int. J. Control, 73:720-732, 2000.

[10] J. J. Gertler and R. Monajemy. Generating directional residuals with dynamic parity relations. Automatica, 31:627-635, 1995.

[11] A. S. Morse. Minimal solutions to transfer matrix equations. IEEE Trans. Autom. Control, 21:131-133, 1976.

[12] Y. Peng, A. Youssouf, Ph. Arte, and M. Kinnaert. A complete procedure for residual generation and evaluation with application to a heat exchanger. IEEE Trans. Control Systems Technology, 5:542-555, 1997.

[13] A. Varga. Computation of irreducible generalized state-space realizations. Kybernetika, 26:89-106, 1989.

[14] A. Varga. On stabilization of descriptor systems. Systems \& Control Letters, 24:133-138, 1995.

[15] A. Varga. Computation of Kronecker-like forms of a system pencil: Applications, algorithms and software. Proc. CACSD'96 Symposium, Dearborn, MI, pp. 77-82, 1996.

[16] A. Varga. Computation of coprime factorizations of rational matrices. Lin. Alg. \& Appl., 271:83-115, 1998.

[17] A. Varga. A descriptor systems toolbox for Matlab. Proc. CACSD'2000 Symposium, Anchorage, Alaska, 2000.

[18] A. Varga. Computing generalized inverse systems using matrix pencil methods. Int. J. of Applied Mathematics and Computer Science, 11:1055-1068, 2001.

[19] S.-H. Wang and E. J. Davison. A minimization algorithm for the design of linear multivariable systems. IEEE Trans. Autom. Control, 18:220-225, 1973.

[20] W. A. Wolovich, P. Antsaklis, and H. Elliott. On the stability of solutions to minimal and nonminimal design problems. IEEE Trans. Autom. Control, 22:8894, 1977.

[21] W. M. Wonham. Linear Multivariable Control: a Geometric Approach. Springer Verlag, New York, 1979.

[22] A. Youssouf and M. Kinnaert. Observer based residual generator for singular systems. Proc. of CDC'96, Kobe, Japan, pp. 1175-1180, 1996. 